\title{
Digit ratio (2D:4D) and prenatal/perinatal sex hormones: A response to Manning and Fink (2017)
}

\author{
Gareth Richards, Ph.D. ${ }^{1}$ \\ ${ }^{1}$ Autism Research Centre, Department of Psychiatry, University of Cambridge, UK \\ * Corresponding author address: Autism Research Centre, University of Cambridge, Douglas House, 18b \\ Trumpington Road, Cambridge, CB2 8AH; email: gvr22@medschl.cam.ac.uk
}

\section{This is a pre-publication version of the following article:}

Richards, G. (2017). Digit ratio (2D:4D) and prenatal/perinatal sex hormones: A response to Manning and Fink (2017). Early Human Development, 113, 75-76. https://doi.org/10.1016/j.earlhumdev.2017.09.004

\begin{abstract}
I published a Letter to the Editor of Early Human Development [1] to provide an overview and discussion of the literature examining digit ratio (2D:4D) in relation to sex hormone concentrations measured from amniotic fluid and umbilical cord blood in humans. My reason was that there exist a vast number of papers reporting on 2D:4D as a proxy for prenatal sex hormone exposure, yet relatively few that have established associations with hormones assayed during the prenatal/perinatal periods of development. My conclusion was that although the literature points towards a negative correlation between 2D:4D and prenatal/perinatal androgen exposure, the link remains tenuous, with most reported effects not being statistically significant. Manning and Fink [2] responded to this Letter with three main points: (1) that there are currently six (rather than four) papers relating to 2D:4D and sex hormones measured from umbilical cord blood, (2) that statistically significant correlations between 2D:4D and perinatal androgen levels in males have been reported, and (3) that examination of maternal circulating sex hormones during the first trimester may present an opportunity to better understand how early exposure to sex hormones relates to 2D:4D in humans. I would like to take the opportunity to extend my thanks to Manning and Fink for raising these important points, and to Early
\end{abstract} Human Development for inviting me to write this response. 
I reported on two studies of amniotic fluid [3,4] and four studies of umbilical cord blood $[5,6,7,8]$. Manning and Fink correctly pointed out that there are two additional studies relating to cord blood. At the time of publishing my Letter, I was unaware of the two papers by Mitsui et al. $[9,10]$. However, I had previously contacted the lead author of Manning and Fink to ask whether he knew of any relevant studies that I had not included but he did not inform me of these.

The two papers to which Manning and Fink now call attention report significant associations between 2D:4D and androgen concentrations from cord blood in males. This means my statement "no statistically significant effects have yet been reported in male-only samples" is in fact incorrect. The first paper [9] reported on a sample of school-aged children from Japan (135 males, 159 females), and, whilst controlling for covariates, observed a negative correlation between insulin-like factor 3 (INSL3) and mean 2D:4D in males $(\beta=-0.377, p<$ 0.05). Closer inspection revealed that digit ratios for the left hand (L2D:4D) were significantly correlated with INSL3 $(\beta=-0.414, p=0.0125)$ whereas those for the right hand (R2D:4D) were not. No significant associations were observed between 2D:4D and testosterone, oestradiol, the ratio of testosterone to oestradiol, progesterone, luteinizing hormone, follicle stimulating hormone, or inhibin B in either males or females (or indeed in the whole sample). The second paper [10] reported on a subsample (45 males, 72 females) from the previously reported sample. After controlling for covariates, dehydroepiandrosterone (DHEA) was negatively correlated with mean 2D:4D $(\beta=-0.361, p<0.05)$ in males, but not in females, and no significant correlations were observed for cortisol, cortisone, or androstenedione. Having now considered the findings of these two additional papers, the take-home message from my first Letter remains the same: most associations so far reported between 2D:4D and sex hormone levels measured from amniotic fluid or umbilical cord blood have not been statistically significant.

Although van de Beek et al. [11] concluded that "of all available sources, amniotic fluid seems to be the best candidate to investigate the effects of early fetal androgen exposure." (p. 663), Manning and Fink call into question its relevance in relation to 2D:4D. Their reasoning is that amniocentesis is typically performed in the second trimester, whereas sexual dimorphism in 2D:4D is present by the end of the first trimester. However, the presence of sexual dimorphism at this stage does not necessarily indicate that development of the trait is complete, or that further androgen and/or oestrogen stimulation is not important (see Knickmeyer et al. [12]). 
For instance, it might be that there are several 'critical periods' during gestation in which stimulation from androgens and/or oestrogens contribute to variance in 2D:4D. It is also noted that testosterone measured from amniotic fluid is a strong predictor of a range of sexually dimorphic traits throughout development $[13,14]$. It might therefore be surprising if $2 \mathrm{D}: 4 \mathrm{D}$ were a predictor of sexually dimorphic phenotypic outcomes but was only a weak correlate of testosterone in amniotic fluid. However, this could be consistent with the suggestion that the 'critical period' for development of 2D:4D exists within a larger time-window of neuromuscular and cerebral sexual differentiation [15].

Manning and Fink suggest examining maternal circulating hormones in the first trimester to investigate effects of early androgen exposure on digit ratio. This is based on two studies $[4,16]$ that have observed negative correlations between maternal serum testosterone and their offspring's subsequent 2D:4D ratios. However, as with those of amniotic fluid and cord blood, both studies reported weak correlations, along with null-findings. One study [4] obtained serum samples between 15.7 and 23.6 weeks' gestation $(M=17.2$ weeks $)$, whereas the other [16] reported much greater variability (5-36 weeks, $M=16, S D=8$ ). The first [4] observed a significant negative correlation between maternal circulating testosterone and newborn offspring R2D:4D when males and females were combined ( $n=106, r=-0.2, p=0.04$ ); although associations in each sex were in the negative direction for each hand, none were statistically significant. The second study [16] observed a negative correlation between maternal circulating testosterone and L2D:4D $(n=89$, rho $=-0.28, p<0.05)$ and a nonsignificant trend in the same direction for R2D:4D. Importantly, this study did not report separate analyses for males and females, and maternal testosterone levels were not predictive of social communication and emotion recognition in their children, even though similar outcomes have been predicted by testosterone assayed from amniotic fluid [13].

Assuming that $2 \mathrm{D}: 4 \mathrm{D}$ is determined during the first trimester, that maternal circulating androgens do directly affect foetal development, and that Manning and Fink are correct in their suggestion that hormone levels measured from amniotic fluid and cord blood "include echoes of hormonal levels from the 1st trimester", it can be predicted that correlations between maternal circulating testosterone and offspring 2D:4D will be stronger when hormones are sampled in the first trimester compared to the second or third. This hypothesis can be tested by collecting maternal serum samples at various stages of pregnancy. If pursuing this line of research, it would be worthwhile to follow a cohort longitudinally, so that the predictive value 
of maternal circulating sex hormone concentrations on 2D:4D can be assessed throughout development. This is particularly important considering that there appears to be a certain amount of lability in 2D:4D across the lifespan [4,12,17,18].

Manning and Fink conclude that they "do not think the links between human 2D:4D and foetal sex hormones are tenuous.", and that "The available evidence is consistent with a real association for both males and females." Although I agree that the general trend of results from studies of amniotic fluid and umbilical cord blood point towards there being a negative correlation between 2D:4D and early androgen exposure, it remains undeniable that most effects reported so far have not been statistically significant. This is the case for androgens and oestrogens sampled from amniotic fluid, umbilical cord blood, and maternal circulation, for digit ratios of the right hand and left hand, and for males as well as females. These observations can be interpreted in several ways. One such explanation is that researchers may have been examining hormonal exposure during gestational time-points at which androgens and/or oestrogens do not exert maximal effects on 2D:4D, and/or that they have been using inappropriate techniques to do so. For instance, it might be that hormones measured from amniotic fluid, umbilical cord blood, or maternal serum do not well reflect foetal circulating levels. It also might be that sensitivity to androgens and/or oestrogens plays a large role in determining 2D:4D, and that studies that examine correlations with absolute levels of hormone concentrations do not address this issue. Equally, it might be that the relationships between 2D:4D and prenatal sex hormone exposure are more complicated, weaker, or less reliably detectable than initially thought.

\section{References}

[1] G. Richards, What is the evidence for a link between digit ratio (2D:4D) and direct measures of prenatal sex hormones? Early Hum. Dev. (2017) (in press), http://dx.doi.org/10.1016/j.earlhumdev.2017.08.003.

[2] J.T. Manning, B. Fink, Are there any "direct" human studies of digit ratio (2D:4D) and measures of prenatal sex hormones? Early Hum. Dev. (2017) (in press), https://doi.org/10.1016/j.earlhumdev.2017.09.003. 
[3] S. Lutchmaya, S. Baron-Cohen, P. Raggatt, R. Knickmeyer, J.T. Manning, 2nd to 4th digit ratios, fetal testosterone and estradiol, Early Hum. Dev. 77 (2004) 23-28, http://dx.doi.org/10.1016/j.earlhumdev.2003.12.002.

[4] T. Ventura, M.C. Gomes, A. Pita, M.T. Neto, A. Taylor, Digit ratio (2D:4D) in newborns: influences of prenatal testosterone and maternal environment, Early Hum. Dev. 89 (2013) 107112, http://dx.doi.org/10.1016/j.earlhumdev.2012.08.009.

[5] R. Çetin, M. Can, E. Özcan, The relationship between testosterone and oestrogen level of the cord blood and length of fingers of newborns 2d:4d, Balikesir Health Sci. J. 5 (2016) 75-82, http://dx.doi.org/10.5505/bsbd.2016.49469.

[6] M. Hickey, D.A. Doherty, R. Hart, R.J. Norman, E. Mattes, H.C. Atkinson, D.M. Sloboda, Maternal and umbilical cord androgen concentrations do not predict digit ratio (2D:4D) in girls: a prospective cohort study, Psychoneuroendocrinology 35 (2010) 1235-1244, http://dx.doi.org/10.1016/j.psyneuen. 2010.02.013.

[7] L.P. Hollier, J.A. Keelan, E.S.L. Jamnadass, M.T. Maybery, M. Hickey, A.J.O. Whitehouse, Adult digit ratio (2D:4D) is not related to umbilical cord androgen or estrogen concentrations, their ratios or net bioactivity, Early Hum. Dev. 91 (2015) 111-117, http://dx.doi.org/10.1016/j.earlhumdev.2014.12.011.

[8] A.J.O. Whitehouse, S.Z. Gilani, F. Shafait, A. Mian, D.W. Tan, M.T. Mayberry, J.A. Keelan, R. Hart, D.J. Handelsman, M. Goonawardene, P. Eastwood, Prenatal testosterone exposure is related to sexually dimorphic facial morphology in adulthood, Proc. R. Soc. B Biol. Sci. 282 (2015) 20151351, http://dx.doi.org/10.1098/rspb.2015.1351.

[9] T. Mitsui, A. Araki, H. Goudarzi, C. Miyashita, S. Ito, S. Sasaki, T. Kitta, K. Moriya, K. Cho, K. Morioka, R. Kishi, N. Shinohara, M. Takeda, K. Nonomura, Effects of prenatal Leydig cell function on the ratio of the second to fourth digit lengths in school-aged children, PLoS One 10 (2015) e0120636, , http://dx.doi.org/10.1371/journal.pone.0120636.

[10] T. Mitsui, A. Araki, H. Goudarzi, C. Miyashita, S. Ito, S. Sasaki, T. Kitta, K. Moriya, K. Cho, K. Morioka, R. Kishi, N. Shinohara, M. Takeda, K. Nonomura, Effects of adrenal androgens during the prenatal period on the second to fourth digit ratio in school-aged children, Steroids 113 (2016) 46-51, http://dx.doi.org/10.1016/j.steroids.2016.06.009.

[11] C. van de Beek, J.H.H. Thijssen, P.T. Cohen-Kettenis, S.H.M. van Goozen, J.K. Buitelaar, Relationships between sex hormones assessed in amniotic fluid, and maternal and umbilical cord serum: what is the best source of information to investigate the effects of fetal hormonal exposure? Horm. Behav. 46 (2004) 663-669, http://dx.doi.org/10.1016/j.yhbeh.2004.06.010. 
[12] R.C. Knickmeyer, S. Woolson, R.M. Hamer, T. Konneker, J.H. Gilmore, 2D:4D ratios in the first 2 years of life: stability and relation to testosterone exposure and sensitivity, Horm. Behav. 60 (2011) 256-263, http://dx.doi.org/10.1016/j.yhbeh.2011.05.009.

[13] B. Auyeung, M.V. Lombardo, S. Baron-Cohen, Prenatal and postnatal hormone effects on the human brain and cognition, Pflugers Arch. - Eur. J. Physiol. 465 (2013) 557-571, http://dx.doi.org/10.1007/s00424-013-1268-2.

[14] S. Baron-Cohen, B. Auyeung, B. Nørgaard-Pedersen, D. Hougaard, M. Abdallah, L. Melgaard, A. Cohen, B. Chakrabarti, L. Ruta, M. Lombardo, Elevated fetal steroidogenic activity in autism, Mol. Psychiatry 20 (2015) 369-376, http://dx.doi.org/10.1038/mp.2014.48.

[15] A. Szwed, M. Kosinska, J.T. Manning, Digit ratio (2D:4D) and month of birth: a link to the solstitial-melatonin-testosterone effect, Early Hum. Dev. 104 (2017) 23-26, http://dx.doi.org/10.1016/j.earlhumdev.2016.11.005.

[16] M. Barona, R. Kothari, D. Skuse, N. Micali, Social communication and emotion difficulties and second to fourth digit ratio in a large community-based sample, Mol. Autism 6 (2015) 68, http://dx.doi.org/10.1186/s13229-015-0063-7.

[17] G. Richards, W. Bellin, W. Davies, Familial digit ratio (2D:4D): associations in a general population sample from Wales, Early Hum. Dev. 112 (2017) 14-19, http://dx.doi.org/10.1016/j.earlhumdev.2017.06.006.

[18] R.L. Trivers, J.T. Manning, A. Jacobson, A longitudinal study of digit ratio (2D:4D) and other finger ratios in Jamaican children, Horm. Behav. 49 (2006) 150-156, http://dx.doi.org/10.1016/j.yhbeh.2005.05.023. 